\section{IJ INDEX PAR AUTEURS}

Anglard P. Voir Louis J.C.

Ardaillou R. Hormones, médiateurs et glomérules. 2: 178-as.

Arnauld E. Voir Vincent J.D.

Arrang J.M. Voir Pollard H.

Aumont M.C. Voir Gourgon R.

Aupetit B. Voir Cabrol C.

Aurias A. Aspects cytogénétiques de

I'ataxie-télangiectasie. 2 : 298-as.

Babinet C. Animaux transgéniques : une voie nouvelle pour l'étude du développement. 2 : 253-as.

Bach J.F. Voir Potworowski E.

Barjon J.N. Voir Bichet D.

Baud F. Voir Gandjbakhch I.

Baumelou A. La pharmacovigilance, pour

quoi faire et comment ? 2 : 395-hd.

Baumelou E. Voir Baumelou A.

Beaud G. Vers un renouveau du virus de

la vaccine. 2 : 94-as.

Beaune P. Les cytochromes P. 450 hépa-

tiques humains. 2 : 358-as.

Bellucci S. Voir Maclouf J.

Benhamou J.P. Métabolisme hépatique des

médicaments et lésions hépatiques médicamenteuses. 2 : 356-ed.

Bensussan $A$. Le récepteur spécifique du lymphocyte T. 2 : 296-ed.

Berger R.: Cytogénétique et cancer. 2 : 246-as.

Berger R. Voir Larsen JC.

Bernard J. Évolution de la bio-éthique. 2 : 480-ed.

Bernard O. Voir Larsen J.C.

Bernheim A. Voir Larsen J.C.

Besson J.M. Les systèmes de contrôle de la douleur. 2: 493-as.

Bichet D. Hypersécrétion de l'hormone antidiurétique chez l'homme. 2: 193-as;

Bismuth V. Voir Lacombe P.

Blanchet F. Voir Nitenberg $A$

Bonvalet J.P. Voir Farman N.

Bourel M. Voir Brissot $P$.

Brissot $P$. Aspects récents de l'hémochro-

matose idiopathique. 2 : 542-as.

Brunette M.G. Transport du phosphore inorganique par le tube proximal du rein. 2: 422-as.

Cabrol A. Voir Cabrol C.

Cabrol C. Transplantation cardiaque : les raisons du succès. $2: 81$-as.

Cabrol C. Voir Gandjbakhch I.

Caillaud $F$. Thrombolytiques : la génération des chimères. 2 : 568-as.

Caster L. Voir Gandjbakhch I.

Caubet J.F. Voir Larsen J.C.

Chandebois R. Débat autour de la notion

\section{ABRÉVIATIONS}

ed : éditorial

as : article de synthèse

hd: hypothèse et débat

no : note originale

It : lettre à $\mathrm{m} / \mathrm{s}$

do : dossier de " programme génétique de développement ". 2 : 514-hd.

Charpentier B. Voir Touraine J.L.

Christel P. Biomatériaux et chirurgie orthopédique. 2 : 573-as.

Cocito C. Archaebactéries et évolution. 2 : 436-as.

Corvol P. De la structure de la rénine à la conception d'inhibiteurs. 2 : 385-as.

David V. Voir Bensussan A.

Dei-Cas E. Physiopathologie du paludisme. 2 : 322-as.

Delaisi de Parseval G. Le statut de l'enfant procréé artificiellement : disparités interna-

tionales. 2 : 482-as.

Delcour A. Voir Cabrol C.

Desbleds M.T. Voir Grenier P.

Deugnier $Y$. Voir Brissot $P$.

Eugène $M$. Voir Lechat $P$.

Fafadet J. Voir Gandbakhch I.

Fagot A. Voir Delaisi de Parseval G.

Farman N. Mécanismes d'action de l'aldostérone dans le rein. 2 : 186-as.

Faure N. Voir Lemay A

Flamand A. Actualités de la rage. 2 : 312-as.

Foster D.W. Voir Lacombe P.

Foult J.M. Voir Nitenberg A.

Frézal J. Anomalies du développement, facteurs de croissance et oncogènes. 2 : 236-ed.

Fries D. Voir Touraine J.L.

Gandjbakhch I. Angioplastie ou chirurgie dans les lésions coronaires. 2 : 99-hd.

Gandjbakhch I. Voir Cabrol C.

Garbarg M. Voir Pollard H.

Gascon-Barré M. La Souris Hyp, un modèle

d'étude de la réabsorption rénale du phos-

phore et de la synthèse de vitamine D. 2 :

420-ed.

Glorieux J. Le développement mental des hypothyroïdiens congénitaux : le succès du dépistage. 2 : 272-no.

Glowinski J. Neurosciences : progrès et perspectives. 2 : 8-ed.

Goltzman D. Le phosphore influence-t-il le métabolisme de la parathormone? 2 : 501-as.

Gourgon R. Traitement de l'insuffisance cardiaque : quel avenir ? 2 : 64-ed.

Grenier P. L'imagerie par résonance magnétique se substituera-t-elle à la tomodensitométrie ? 2 : 130-as.

Guilbeau J.C. Voir Grenier P.

Hourmant M. Voir Touraine J.L.

Jacques $Y$. Voir Touraine J.L.

Jaffrin M.Y. Voir Reach G.

Jault F. Voir Gandjbakhch I.

Jouannet $P$. Problèmes éthiques soulevés par la congélation d'embryons humains. 2 : 345-do.

Junien C. Les antioncogènes. 2 : 238-as. Kahn A. médecine/sciences, ses lecteurs, ses projets. 2 : 538-ed.

Komajda M. Voir Lechat $P$

Kreis H. Voir Touraine J.L.

Kutten F. Voir Mauvais-Jarvis $P$.

Lacombe P. Principes, applications et perspectives de l'imagerie radiologique numérique. 2 : 144-as.

Langlois $A$. Les comités d'éthiques : situation canadienne, débat français. 2 : 489-as.

Larrey D. Polymorphisme génétique du métabolisme hépatique des médicaments. 2 : 364-as.
Larsen J.C. Voir Berger R.

Larsen J.C. Réarrangements des gènes cmyc et Tc $R-\alpha$ dans les leucémies et lymphomes T. 2 : 295-It.

Lechat P. Mécanismes de la contractilité cardiaque. 2 : 73-as.

Lefranc G. Voir Lefranc M.P.

Lefranc M.P. Délétion de gènes de la région constante des chaînes lourdes des immunoglobulines humaines. 2 : 508-as. Léger P. Voir Cabrol C.

Lemay A. Les agonistes de la LHRH en gynécologie. 2 : 454-as.

Lerch R. Voir Ratib 0 .

Lompre A.M. Voir Schwartz K

Lorphelin J.M. Échotomographie : les nouveaux champs d'application. 2 : 127-as. Louis J.C. Rôle de I'ACTH dans la protection des neurones contre l'anoxie. 2 : 45-no.

Maclouf J. Thromboxane A2, Prostacycline ou le duel plaquettes-vaisseaux. 2 : 552-as.

Maheux R. Voir Lemay A.

Malissen B. Voir Malissen M.

Malissen M. Réarrangements somatiques des gènes du récepteur des lymphocytes T. 2 : 304-as.

Marco J. Voir Gandjbakhch

Marguerie G. Le fibrinogène, facteur multifonctionnel de l'hémostase. 2 : 260-as. Martin N. L'imagerie par résonance magnétique en neuroradiologie. 2 : 137-as. Mathieu-Mahul D. Voir Larsen J.C.

Mattei M.F. Voir Cabrol C.

Mattei M.G. L'hybridation in situ en cytogénétique. 2 : 35-as.

Mauchauffé M. Voir Larsen J.C.

Maurois P. Voir Dei-Cas E.

Mauvais-Jarvis $P$. L'androgénisation humaine: rôle d'une enzyme clé. 2 : 267-as.

Ménard J. Voir Corvol P.

Menu Y. Caractérisation tissulaire en échotomographie. Application à la pathologie hépatique. 2 : 122-as.

Mercadier J.J. Voir Schwartz K.

Moreau J.F. Voir Touraine J.L.

Morello D. Voir Babinet C.

Mouvier M. Voir Lacombe $P$.

Mowszowicz I. Voir Mauvais-Jarvis P.

Nahum H. Les nouvelles méthodes d'imagerie en 1986. 2 : 120-ed.

Nitenberg A. Sarcoïdose cardiaque et vasoconstriction chronique de la microcirculation coronaire. 2 : 211-no.

Papukna V. Voir Bichet D.

Pavie A. Voir Cabrol C.

Pavie A. Voir Gandjbakhch I.

Perrenec J. Voir Nitenberg A

Pessayre $D$. Mécanismes des hépatites médicamenteuses. 2 : 373-as.

Petitbon P. Voir Lacombe P.

Pollard $\mathrm{H}$. Les transmissions histaminergiques cérébrales. 2 : 10-as.

Potworowski E. Vers un thymus artificiel ? 2 : 331-hd.

Ratib O. Tomographie à émission de positrons et exploration du myocarde. 2 : 445-as.

Reach G. Les pancréas bioartificiels. 2 : 87-as.

Renard J.P. La congélation de l'embryon humain. 2 : 26-as.

Roques B.P. Pharmacologie moléculaire des neuropeptides. 2 : 17-as. 
Rosa J. Un modèle en recherche clinique : la drépanocytose. 2 : 558-as. Rossier B. Voir Farman N. Rottembourg J. Voir Cabrol C.

Rouffignac (de) C. Rein et hormones : une régulation complexe. 2 : 176-ed. Roujeau J.C. Le syndrome de Lyell. 2 : 380-as.

Schwartz J.C. Voir Pollard $\mathrm{H}$. Schwartz K. Génétique moléculaire de I'hypertrophie cardiaque. 2 : 66-as.

Sigaux F. Voir Larsen J.C.

Simon M. Voir Brissot P.

Soulillou J.P. Voir Touraine J.L.

Tellier P. Voir Nitenberg A.

Tenenhouse H.S. Rachitisme hypophospha-

témique héréditaire. 2 : 429-as.

Touraine J.L. Le rejet d'allogreffe : les nouveaux thérapeutes ont-ils la panacée ? 2 206-hd.

Valeyre D. Voir Nitenberg A.

Verderi D. Voir Lacombe P.

Vernes A. Voir Dei-Cas E.

Vincendon G. Voir Louis J.C.

Vincent J.D. Le cerveau hormonal. 2 : 198-as.

Wright F. Voir Mauvais-Jarvis $P$.

Zarifian E. En quête d'index biologiques des maladies mentales. 2 : 42-hd.

\section{INDEX PAR SUJETS}

\section{Bioesthique}

Les comités d'éthique : situation canadienne-débat français. 2 : 489-as. Évolution de la bio-éthique. 2 : 480-ed. Le statut de l'enfant procréé artificiellement : disparités internationales. 2 : 482-as.

\section{Cardiologie-Hémostase}

Angioplastie ou chirurgie dans les lésions coronaires. 2 : 99-hd.

Le fibrinogène, facteur multifonctionnel de l'hémostase. 2 : 260-as.

Génétique moléculaire de l'hypertrophie cardiaque. 2 : 66-as.

Mécanismes de la contractilité cardiaque. 2 : 73-as.

Sarcoïdose cardiaque et vasoconstriction chronique de la microcirculation coronaire. 2: 211-no.

Thrombolytiques : la génération des chimères. 2 : 568-as.

Thromboxane $A_{2}$, Prostacycline ou le duel plaquettes - vaisseaux. 2 : 552-as.

Traitement de l'insuffisance cardiaque : quel avenir ? 2 : 64-ed.

Transplantation cardiaque : les raisons du succès. 2 : 81 -as.

Chirurgie

Biomatériaux et chirurgie orthopédique. 2 : 573-as.

Dermatologie

Le syndrome de Lyell. 2 : 380-as.

Génétique-Oncogènes-Développement

Animaux transgéniques : une voie nouvelle

pour l'étude du développement. 2 :

253-as.

$\mathrm{m} / \mathrm{s} n^{\circ} 10$ ool. 2, décembre 86
Anomalies du développement, facteurs de croissance et oncogènes. 2 : 236-ed. Les antioncogènes. 2 : 238-as.

Aspects cytogénétiques de l'ataxietélangiectasie. 2 : 298-as.

La congélation de l'embryon humain. 2 : 26-as.

Cytogénétique et cancer. 2 : 246-as.

Débat autour de la notion de " programme génétique de développement ". 2 : 514-hd. L'hybridation in situ en cytogénétique. 2 : 35-as.

Un modèle en recherche clinique : la drépanocytose. 2 : 558-as.

Problèmes éthiques soulevés par la congélation d'embryons humains. 2 : 345-do.

Réarrangements des gènes $c$-myc et Tc R-

$\alpha$ dans des leucémies et lymphomes T. 2 : 295-It.

\section{Hépatologie-Médicaments}

Aspects récents de l'hémochromatose idiopathique. 2 : 542-as.

Les cytochromes P-450 hépatiques humains. 2 : 358-as.

Mécanismes des hépatites médicamenteuses. 2 : 373-as.

Métabolisme hépatique des médicaments et lésions hépatiques médicamenteuses. 2 . 356-ed.

La pharmacovigilance, pour quoi faire et comment ? 2 : 395-hd.

Polymorphisme génétique du métabolisme hépatique des médicaments. 2 : 364-as. Imagerie médicale

Caractérisation tissulaire en échotomographie. Application à la pathologie hépatique. 2 : 122-as.

Échotomographie : les nouveaux champs d'application. 2 : 127-as.

L'imagerie par résonance magnétique en neuroradiologie. 2 : 137-as.

L'imagerie par résonance magnétique se substituera-t-elle à la tomodensitométrie 2 : 130-as.

Les nouvelles méthodes d'imagerie en 1986. 2 : 120-ed.

Principes, applications et perspectives de l'imagerie radiologique numérique. 2 : 144-as.

Tomographie à émission de positrons et exploration du myocarde. 2 : 445-as. Immunologie

Délétion de gènes de la région constante des chaînes lourdes des immunoglobulines humaines. 2 : 508-as.

Réarrangements somatiques des gènes du récepteur des lymphocytes T. 2 : 304-as Le récepteur spécifique du lymphocyte $T$. 2 : 296-ed.

Vers un thymus artificiel ? 2 : 331-hd.

Maladies infectieuses, Bactériologie, Virologie, Parasitologie

Actualité de la rage. 2 : 312-as.

Archaebactéries et évolution. 2 : 436-as. Physiopathologie du paludisme. 2 : 322-as. Vers un renouveau du virus de la vaccine. 2 : 94-as.

Néphrologie, Endocrinologie

Les agonistes de la LHRH en gynécologie. 2 : 454-as.

L'androgénisation humaine: rôle d'une enzyme clé. 2 : 267-as.

Le développement mental des hypothyroïdiens congénitaux : le succès du dépistage. 2 : 272-no.
Hormones, médiateurs et glomérules. 2 : 178-as.

Hypersécrétion de l'hormone antidiurétique chez l'homme. 2 : 193-as.

Mécanismes d'action de l'aldostérone dans le rein. 2 : 186-as.

Les pancréas bioartificiels. 2 : 87-as.

Le phosphore influence-t-il le métabolisme de la parathormone. 2 : 501-as.

Rachitisme hypophosphatémique héréditaire. 2 : 429-as.

Rein et hormones : une régulation complexe. 2 : 176-ed.

Le rejet d'allogreffe : les nouveaux thérapeutes ont-ils la panacée? 2 : 206-hd.

Souris Hyp, un modèle d'étude de la réabsorption rénale du phosphore et de la synthèse de vitamine D. 2 : 420-ed. De la structure de la rénine a la conception d'inhibiteurs. 2 : 385-as.

Transport du phosphore inorganique par le tubule proximal du rein. $2: 422$-as.

Neurosciences

Le cerveau hormonal. 2 : 198-as.

Neurosciences : progrès et perspectives; 2 ? 8-ed.

Pharmacologie moléculaire des neuropeptides. 2 : 17-as.

En quête $d$ 'index biologiques des maladies mentales. 2 : 42-hd.

Rôle de I'ACTH dans la protection des neurones contre l'anoxie. 2 : 45-no.

Les systèmes de contrôle de la douleur. 2 : 493-as.

Les transmissions histaminergiques cérébrales. 2 : 10-as.

\section{DO
NOUVELEX}

Biologie générale et médicale

La cachectine. J.-C.D. $2: 49$.

Un agent fibrinolytique spécifique. J.-C.D. 2 : 49

Les enzymes des lysosomes et leur pathologie. J.-C.D. 2 : 52.

Nous avons l'åge de nos neurones. A.K. $2: 53$.

Localisation de la zone épileptogène. J.C.D. 2 : 107.

Des anticorps contre I'acétylcholine J.-C.D. 2 : 154.

Sclérose en plaques et rétrovirus de la famille HTLV. A.K. 2 : 155

Un facteur érythropoḯtique et une antiprotéase sont-ils identiques? J.-C.D. $2: 217$. Fonctionnement autocrine des cellules et cancer. A.K. 2 : 220.

Localisation cérébrale des monoamine oxydases A et B. J.-C.D. $2: 222$.

Maladie d'Alzheimer et neuropeptides (suite). J.-C.D. 2 : 277.

Mais à quoi sert donc l'ubiquitine ? A.K. 2 : 282.

Les endosomes. J.-C.D. $2: 334$.

La bursine, hormone de différenciation des lymphocytes B aviaires est un tripeptide. A.K. 2 : 335.

Un signal polyvalent de reconnaissance cellulaire. J.-C.D. 2 : 337. 
Transport des protéines vers et à travers les membranes. A.K. 2 : 341.

Des scientifiques philosophes. F.S. 2 : 342.

Le G.R.F. J.-C.D. 2 : 344.

Un modèle de maladie de Huntington induite chez le rat par l'acide quinolinique. J.-C.D. $2: 400$.

Thymus et cerveau. A.K. 2 : 401.

AMP cyclique, apprentissage et mémoire.

A.K. $2: 407$

Benzodiazépines et rythmes circadiens. J.C.D. 2 : 411

Mécanisme d'action de l'insuline et de son récepteur. A.K. 2 : 464.

Inhibine, FRP et activine. A.K. $2: 466$.

Les prions : essor, décadence, renaissance. J.-C.D. 2 : 588.

Un peptide de synthèse inhibe les métastases du mélanome de souris. J.-C.D. 2 : 586.

Biologie moléculaire

Souris transgéniques et virus de l'hépatite B. A.K. $2: 48$.

Génétique et particules d'information, redistribution des exons au cours de l'évolution. A.K. 2 : 51.

Angiogénine. J.-C.D. 2 : 107.

Hasard ou nécessité : des virus qui se ressemblent. A.K. 2 : 110.

Le récepteur des glucocorticoïdes et son homologie avec l'oncogène v-erb-A. A.K. 2 : 151 .

L'hyperexpression d'oncogènes cellulaires est vraiment cancérigène in vivo. A.K. 2 : 158.

Réarrangement des gènes codant pour le récepteur des cellules lymphocytaires $T$ au cours des syndromes lymphoprolifératifs. A.K. 2 : 160 .

Et si les introns servaient à quelque chose ? A.K. 2 : 221.

Quand les oncogènes se comportent comme des différenciateurs. A.K. 2 : 222. Des plantes transgéniques. A.K. $2: 223$.

Du nouveau dans la biotechnologie des végétaux. A.K. 2 : 224.

Épissage des ARN et ribozymes. A.K. 2 : 280.

AMP cyclique, protéine kinase et contrôles transcriptionnels. A.K. 2 : 284

La transactivation du virus LAV est traductionnelle. A.K. 2 : 285.

Régulation post-transcriptionnelle de I'expression des oncogènes. A.K. 2 : 286. L'ARN cellulaire peut-il se répliquer ? A.K. 2 : 338.

Visualiser des molécules en action. P.B. 2 : 401.

Mécanismes moléculaires de la vision des couleurs et du daltonisme. J.-C.D. 2 : 402. Un messager du vieillissement. A.K. 2 : 404.

Cancers génitaux et virus du papillome. A.K. $2: 405$.

Un gène dans un gène. A.K. 2 : 408.

Une sonde $A D N c$ spécifique de virus de I'hépatite D. S.E. 2 : 40\$.

Régulation positive et négative des enhancers. A.K. $2: 410$.

Rétrovirus humains et sclérose en plaque.

A.K. 2 : 463.

Mécanismes post-traductionnels d'activa-

Des inhibiteurs de la croissance cellulaire. A.K. 2 : 467

Oncogènes, facteurs de croissance et lipocortines. A.K. 2 : 522.

Les $G$ protéines, un système universel de transduction des signaux. A.K. 2 : 583. Régulation post-transcriptionnelle de la phosphatase alcaline hépatique. S.E. 2 : 587.

Le génome des Papillomavirus, agent probable de cancers et modèle unique de biologie fondamentale. F.C. 2 : 589.

\section{Génétique}

Maladie de Hunter chez 2 filles. J.-C.D. 2 : 53.

Gènes paternels et maternels : différence de gravité des maladies héréditaires. J.C.D. 2 : 103.

Albinisme et voies optiques. J.-C.D. 2 : 106.

Empreintes individuelles d'ADN (suite). J.C.D. 2 : 155

Transmission pseudo-autosomale des séquences d'ADN localisées sur les chromosomes sexuels. A.K. 2 : 157.

La stéroïde sulfatase. J.-C.D. 2 : 159.

Mutations des horloges biologiques, une grande découverte. A.K. 2 : 223.

L'ADN, marqueur d'évolution des populations humaines J.-C.D. $2: 278$.

Clonage et expression d'une protéine humaine anti-inflammatoire. J.-C.D. 2 : 279.

a mouche au secours des embryologistes. A.K. $2: 283$

Clonage d'embryon. A.K. 2 : 335.

Antigène $\mathrm{H}-\mathrm{Y}$ et spermatogenèse. J.-C.D. 2 : 336 .

Atteinte neurologique chez des souris porteuses d'une translocation équilibrée. J.C.D. 2 : 339.

La membrane cellulaire cible de facteurs régulant le développement embryonnaire. A.K. 2 : 340

Une fourmi avec une seule paire de chromosomes. J.-C.D. 2 : 344

Les empreintes d'ADN dans le commerce. J.-C.D. 2 : 401

Le clonage du facteur VII. J.-C.D. 2 : 409.

Non maturation du facteur $I X$, une cause d'hémophilie B. J.-C.D. 2 : 462.

Le locus génétique de la myotonie dystrophique de Steinert. J.-C.D. 2 : 463. Identification du gène de la granulomatose chronique. A.K. $2: 521$

Le gène de la myopathie de Duchenne : la chasse aux exons est ouverte. J.-C.K. 2 : 524.

Génétique moléculaire de deux maladies neurologiques héréditaires de la souris. J.C.D. 2 : 526.

Le déficit en stéroïde 21 hydroxylase mécanismes moléculaires. J.-C.D. 2 : 527. Des mutations ponctuelles a l'origine des amyloses. J,-P. G, A.K. 2 : 528.

Transplantation de cellules souches in utero chez le mouton. J.-C.D. 2 : 582

Localisation précise du gène de l'hémochromatose héréditaire. J.-C.D. 2: 586 .

Clinique et thérapeutique

Un marqueur du rein polykistique. J.-C.D. $2: 49$.

Une nouvelle variété d'amylose. J.-P.G. 2 : 106.
Démographie : état actuel et horizon 2020. J.-C.D. 2 : 108.

Épuration enzymatique de la bilirubine sanguine. J.-C.D. 2 : 156.

Maladie de Creutzfeldt-Jakob et hormone de croissance. J.-C.D. 2 : 220

Restituer la mémoire aux individus ågés ? J.-C.D. 2 : 224.

Le SIDA est-il responsable d'hépatites? S.E. 2 : 275.

Vers la vaccination antipalustre. A.K. 2 : 282.

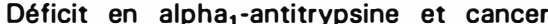
primitif du foie. S.E. $2: 336$.

Ondes de choc contre calculs biliaires. S.E. 2 : 343.

Une utilisation inédite des essais nucléaires : la datation des calculs biliaires. S.E. 2 : 406.

La cyclosporine induit des rémissions du diabète insulino-dépendant. J.-P. G. 2 : 460.

SIDA : variabilité génétique du virus et progression de l'épidémie. A.K. 2 : 461.

SIDA : enfin une drogue semblant active A.K. 2 : 520

Vaccination des animaux sauvages contre la rage. A.K. 2 : 523.

Un essai thérapeutique chez des jumeaux identiques atteints de myopathie de Duchenne. J.-C.D. 2 : 523.

Lexiques

Contrôle hormonal de l'expression des gènes. A.K. 2 : 50.

'hybridation moléculaire, le "Southern blot ". A.K. 2 : 104.

Hybridation moléculaire : les polymorphismes de taille des fragments de restriction. A.K. 2 : 152

Les différents types de mutation d'un gène et leurs conséquences. A.K. 2 : 218.

Mutations des gènes : anomalies d'épissage et décalages de phase de lecture. A.K. 2 : 276

Clonage de gènes. A.K. 2 : 333.

Analyse directe des anomalies de I'ADN chez l'homme. A.K. 2 : 398.

L'analyse de I'ARN en médecine. A.K. 2 : 518.

Génie génétique, production de médicaments et thérapie génique. A.K. 2 : 579.

NOUVELLES réalisées par:

J.-C. Dreyfus,

S. Erlinger

J.-P. Grünfeld,

A. Kahn.

Avec le concours de :

P. Briand,

F. Cuzin.

J.-C. Kaplan,

F. Shapira. 